\title{
Naive Bayes Image Classification: beyond Nearest Neighbors
}

\author{
Radu Timofte ${ }^{1}$, Tinne Tuytelaars ${ }^{1}$, and Luc Van Gool ${ }^{1,2}$ \\ ${ }^{1}$ ESAT-VISICS /IBBT, Catholic University of Leuven, Belgium \\ ${ }^{2}$ D-ITET, ETH Zurich, Switzerland
}

\begin{abstract}
Naive Bayes Nearest Neighbor (NBNN) has been proposed as a powerful, learning-free, non-parametric approach for object classification. Its good performance is mainly due to the avoidance of a vector quantization step, and the use of image-to-class comparisons, yielding good generalization. In this paper we study the replacement of the nearest neighbor part with more elaborate and robust (sparse) representations, as well as trading performance for speed for practical purposes. The representations investigated are $k$-Nearest Neighbors $(k N N)$, Iterative Nearest Neighbors $(I N N)$ solving a constrained least squares (LS) problem, Local Linear Embedding $(L L E)$, a Sparse Representation obtained by $l_{1}$-regularized LS $\left(S R_{l_{1}}\right)$, and a Collaborative Representation obtained as the solution of a $l_{2}$-regularized LS problem $\left(C R_{l_{2}}\right)$. In particular, NIMBLE and K-DES descriptors proved viable alternatives to SIFT and, the $\mathrm{NB} S R_{l_{1}}$ and $\mathrm{NB} I N N$ classifiers provide significant improvements over NBNN, obtaining competitive results on Scene-15, Caltech101, and PASCAL VOC 2007 datasets, while remaining learning-free approaches (i.e., no parameters need to be learned).
\end{abstract}

\section{Introduction}

In [1], Boiman et al. introduced a novel, non-parametric approach for image classification, the Naive Bayes Nearest Neighbor classifier (NBNN). Given an image represented by a set of extracted features, and a priori sets/classes of such features with known label, NBNN is searching for the class to which the query features have the minimum cumulated distance. In spite of being a learning-free method and the lack of tuned parameters, this scheme achieves close to stateof-the-art results for image classification. This is due to: i) the lack of a vector quantization step, avoiding thus to introduce more discretization errors, and ii) the use of image-to-class comparisons instead of image-to-image, which allows to combine bits and pieces of information from multiple training images.

At the same time, NBNN also has its limitations. The most important one is the high computational cost during testing: computation time depends linearly on the number of extracted features from the query image and linearly to logarithmicly on the number of stored labeled features. Unfortunately, the performance heavily depends on the density of features: the more information, the better the results but the slower the evaluation. Some have also criticized 
the Naive Bayes learning free approach, as it needs balanced data with similar density in feature space for all classes, which often is not the case, and also the feature independence assumption is questionable. Learning is the solution to these problems, as addressed by [2-4]. Here we do not follow this strand of work, but rather stick to the simplicity of the original, parameter-free NBNN based on Naive Bayes. Instead, we explore another, complementary direction for improving the results, by investigating alternative representations that can replace the Nearest Neighbor scheme. We believe it is easier to spot the relative power of different representations in a learning-free formulation. Adapting the learningbased extensions of NBNN of [2-4] to the Naive Bayes variants investigated here seems relatively straightforward.

NBNN starts from the basic Naive Bayes (NB) Classifier, and uses the distance to the Nearest Neighbor (NN) as an approximation of the log-probability for a single feature to belong to a certain class of features. This is a somewhat arbitrary choice, yet critical for the overall performance. Arguably, NN is not the best option. In sparse coding approaches for object classification [5], NN in the form of so-called 'hard assignment' was proven inferior to the 'soft-assignment' approaches such as $l_{1}$-regularized least squares. Moreover, the NN classifier has considerably lower performance than state-of-the-art classifiers such as Support Vector Machines or Sparse Representation-based Classifiers (SRC) for the recognition of faces [6], handwritten digits or traffic signs [7].

Replacing the NN with a different representation in the NB classifier formulation is not affecting the desirable properties of NBNN: i) discretization is still avoided, ii) the good generalization of 'image-to-class' is maintained, and iii) it is still a learning free method. The parameters of the representations are adjustable, but usually have known general values for which the performance is reasonably high on (largely) different features and settings.

The main contributions of this paper are as follows. We investigate more general variations of the Naive Bayes Classifier starting from sparse representations. We empirically evaluate $k$-Nearest Neighbors $(k N N)$, Local Linear Embedding (LLE) [8], Iterative Nearest Neighbors (INN) [9], Sparse Representation with $l_{1}$-regularization $\left(S R_{l_{1}}\right)[6]$, and the Collaborative Representation with $l_{2}$-regularization $\left(C R_{l_{2}}\right)$ [10]. Except for the last one, all of these are sparse. Moreover, we use in the NB framework for the first time the recently proposed Kernel Descriptors [11] and compare them to the results obtained with NIMBLE features [12].

Another side contribution is the evaluation of speeding up strategies for Naive Bayes Classification. We show that with only a small overhead over the original NBNN introduced by computing the rich representations, we are able to improve the performance. Moreover, we evaluate schemes with asymmetric sampling density as in [4] and approximated nearest neighbors (ANN) [13], with respect to the impact on performance and the speedup achieved.

Section 2 of this paper reviews the NB classifier and the sparse representations based variants. Section 3 gives a time complexity analysis. Section 4 
describes our experimental results. Section 5 discusses trade-offs between performance and speed for practical applications, and Section 6 concludes the paper.

\section{Naive Bayes classification}

The NB classifier [1] is a probabilistic classifier working under strong independence assumptions. A query image is represented by a set $Q=\{q\}$ of features which are assumed to be independently sampled from a class-specific feature distribution $p(q \mid c)$. Assuming uniform priors $p(c)$, the classification then becomes

$$
\begin{aligned}
& \hat{c}=\arg \max _{c \in C} p(c \mid Q) \quad=\arg \max _{c \in C}\left\{\prod_{q \in Q} p(q \mid c)\right\} \\
& =\arg \min _{c \in C}-\log \left\{\prod_{q \in Q} p(q \mid c)\right\}=\arg \max _{c \in C}\left\{\sum_{q \in Q} \log p(q \mid c)\right\}
\end{aligned}
$$

In the following we review some of the most popular techniques for defining a (sparse) representation for a query from a given set of samples and we show how the Naive Bayes Classifier can be adapted to use these representations.

\section{$2.1 \quad k$ Nearest Neighbors $(k N N)$}

$k N N$ is a standard method for defining the locality of a sample. The parameters required are the similarity or distance measure necessary for ordering the neighbors and the number of nearest neighbors to be kept. Boiman et al. [1] have shown impressive results using $\mathrm{NB}$ and $1 N N$ for image classification.

In the case of NB- $N N_{k}$, for a query image represented by its set of features $Q=\{q\}$, we compute the $k$-nearest neighbors from each class $c \in C$, notated $N_{k}^{c}(q)$, and the (squared) distance-to-class $d_{q}^{c}$ and the classification becomes

$$
\hat{c}=\arg \min _{c \in C} \sum_{q \in Q} d_{q}^{c}, \quad d_{q}^{c}=\sum_{x \in N_{k}^{c}(q)}\|q-x\|^{2}
$$

which corresponds to the class with the minimum cumulated distances to the query. It is assumed that $p(q \mid c) \sim \exp \left(-d_{q}^{c}\right)$. Here, we consider only neighborhood sizes: $k=1$, i.e. $\mathrm{NBN} N_{1}$, which is the original NBNN [1] and $k=5$, referred to as $\mathrm{NBN} N_{5}$.

\subsection{Local Linear Embedding ( $L L E)$}

LLE [8] was introduced as a non-linear dimensionality reduction method, and recently incorporated in the Locality-constrained Linear Coding (LLC) method [14].

For a given sample $q$ and the set $N_{k}(q)$ of $k N N$ from all the labeled samples $X$, LLE solves:

$$
\min _{w}\left\|q-\sum_{x \in N_{k}(q)} w_{x} x\right\|^{2}, \quad \text { subject to }\|w\|_{1}=1
$$

where $w$ is a $k$-dimensional weights vector corresponding to the $k N N$, with its $l_{1}$-norm constrained to 1 . In our experiments, we consider $5 N N$ as in LLC [14]. 
The importance of each sample $x \in N_{k}(q)$ in the reconstruction of $q$ is given by the absolute value of the assigned weight, $\tilde{w}_{x}=\left|w_{x}\right|$, while the nonneighboring samples, $x \notin N_{k}(q)$, get $\tilde{w}_{x}=0$. We further $l_{1}$-normalize the importance weights for a sample $q$ to sum to 1 , yielding $\hat{w}^{q}=\tilde{w} /\|\tilde{w}\|_{1}$. Now the likeliness of $q$ to belong to class $c$ is given by:

$$
d_{q}^{c}=\sum_{x \in X_{c}} \hat{w}_{x}^{q}
$$

where $X_{c}$ is the subset of $X$ corresponding to the class $c$.

The decision for the NBLLE classifier gives the class with the largest importance/likeliness in locally linear embedding the query image $Q$ :

$$
\hat{c}=\arg \max _{c \in C} \sum_{q \in Q} d_{q}^{c}
$$

\subsection{Sparse Representation with $l_{1}$-min $\left(S R_{l_{1}}\right)$}

The power of $l_{1}$-regularized least squares or lasso is proven for problems such as face recognition [6] or dimensionality reduction [7]. Given the set of $M$ labeled samples $X=\{x\}$, a matrix is formed $\mathbf{X}=\left[x_{1}, x_{2}, \ldots, x_{M}\right]$. Then, for a given sample $q$, we optimize over $w$ (we fix $\lambda=0.3$ ):

$$
\min _{w}\|q-\mathbf{X} w\|_{2}+\lambda\|w\|_{1}
$$

For capturing the importance of the weights for the query $q$, we again take their absolute value $\tilde{w}=|w|$, as in [7], and further $l_{1}$ normalize them as in the case of $L L E$, yielding $\hat{w}^{q}=\tilde{w} /\|\tilde{w}\|_{1}$. Now the likeliness $d_{q}^{c}$ of $q$ to belong to class $c$ and the decision for the $\mathrm{NBS} R_{l_{1}}$ classifier is taken similarly to $\mathrm{NB} L L E$ (eqs. (4)(5)) and selects the class that scores the largest importance/likeliness in the sparse representations of the query image $Q$. In this form $\mathrm{NB} S R_{l_{1}}$ has been used in [9].

\subsection{Collaborative Representation $\left(C R_{l_{2}}\right)$}

$C R_{l_{2}}$ [10] seen as $l_{2}$-regularized least squares can be as good as the $S R_{l_{1}}$ on face recognition under specific conditions, like high dimensional face representations and suitable features such as eigenfaces. A strong point of this approach is the existence of an algebraic solution for

$$
\min _{w}\|q-\mathbf{X} w\|_{2}^{2}+\lambda\|w\|_{2}^{2}
$$

(we fix $\lambda=0.001$ ) such that the coding of $q$ over $X$ is given by

$$
w=\left(\mathbf{X}^{T} \mathbf{X}+\lambda \mathbf{I}\right)^{-1} \mathbf{X}^{T} q
$$

For a specific class $c$ the regularized residuals are computed, as in [10]:

$$
r_{c}^{q}=\left\|q-\mathbf{X}_{c} w_{c}\right\|_{2} /\left\|w_{c}\right\|_{2}
$$


where $w_{c}$ is the (sub)vector of coefficients corresponding to the class $c$ samples from the whole representation $w$. Similarly $\mathbf{X}_{c}$ is the (sub)matrix of $\mathbf{X}$ containing only the samples corresponding to class $c$. The decision for $\mathrm{NBC} R_{l_{2}}$ is then

$$
\hat{c}=\arg \min _{c \in C} \sum_{q \in Q} r_{q}^{c}
$$

corresponding to the class with minimum cumulated residuals to the query $Q$.

\subsection{Iterative Nearest Neighbors (INN)}

$I N N$ [9] representation is an approximate solution to a LS decomposition with imposed coefficients/weights and optimized selected samples. INN tries to fill the gap between fast $k N N$ approaches and powerful but slow sparse or collaborative representations $\left(S R_{l 1}, C R_{l 2}\right)$. Given the set of labeled samples $X=\{x\}$, $k$ samples $s_{i}$ are picked iteratively optimizing

$$
\arg \min _{\left\{s_{i}\right\}_{i=1}^{K}}\left\|q-\sum_{i=1}^{K} \frac{\lambda}{(1+\lambda)^{i}} s_{i}\right\|_{2}, \quad K=\left\lceil-\frac{\log (1-\beta)}{\log (1+\lambda)}\right\rceil
$$

where $\lambda$ is set to 0.1 and $\beta$ is 0.95 as in [9], thus $K=25$. Since the weights are nonnegative and sum up to 1 , the likeliness of $q$ to belong to class $c$ is taken as:

$$
d_{q}^{c}=\sum_{s_{i} \in X_{c}} w_{s_{i}}^{q}=\sum_{s_{i} \in X_{c}} \frac{\lambda}{(1+\lambda)^{i}}
$$

where $X_{c}$ is the subset of $X$ corresponding to the class $c$ and $s_{i}$ is the $i$-th selected sample in the $I N N$ representation.

The decision for the NBINN classifier is taken similarly to NBLLE (eq. (5))

\section{Time analysis}

\subsection{Time complexity analysis}

$\operatorname{NB} N N_{k}$ depends on the number of features from the query image, $N=|Q|$, the number of labeled features, $M=|X|$, the dimensionality of the features, $D$, and the number of nearest neighbors, $k$. The time complexity of a straightforward implementation is $O(N M D+N M k)$. Using the structure of the known data, and organizing it with standard data structures such as kd-trees or hashing tables, allows for a practical sub-linear search in $M$ for $k$ nearest neighbors, without loss of accuracy. However, the higher the dimensionality of the features, the lower the speedup achieved, to the point of no gain.

$\mathrm{NB} L L E$ has a time complexity of $O\left(N M D+N M k+N M k^{2} D\right)$, where $O\left(N M k^{2} D\right)$ is the added time complexity introduced by solving the local linear embedding over $k$-nearest neighbors. In most practical cases $k \ll D$, thus $\mathrm{NB} L L E$ is $k^{2}$ times slower than $\mathrm{NB} N N_{k}$. 
$\mathrm{NB} I N N$ is running iteratively $N N$, thus its time complexity is the number $K$ of iterations, equation (11), times the time complexity of $\mathrm{NBN} N_{1}, O(N M D K)$.

$\mathrm{NBS} R_{l_{1}}$ greatly depends on the $l_{1}$-minimization solver employed for solving equation (6). The feature sign algorithm solver used by us [15] has a time complexity, in the optimal case, of $O(M D s)$, where $s$ depends on the non-zero coefficients in the solution. The solutions tend to be very sparse, so $s$ is small. The time complexity of NBS$R_{l_{1}}$ is $O(N M D s)$ and, still, much slower than $\mathrm{NB} N N_{k}$.

For $\mathrm{NBC} R_{l_{2}}$, first computing the projection matrix of equation (8) greatly reduces the further computation time. The time complexity for this is $O\left(M^{2} D+\right.$ $\left.M^{3}+N M D\right)$, so depending heavily on the number of labeled features, $M$. If one uses the pseudoinverse [16] then we have $O\left(D^{3}+D^{2} M+N M D\right)$.

With naive implementations all the NB variants presented are adding time complexity to that of the original $\mathrm{NBN} N_{1}$ algorithm. Usually, in our settings, the running times are, from the fastest to the slowest, in the following order: $\mathrm{NB} N N_{1}<\mathrm{NB} N N_{k}<\mathrm{NB} L L E \approx \mathrm{NB} I N N \ll \mathrm{NB} S R_{l_{1}}<\mathrm{NBC} R_{l_{2}}$, where $\mathrm{NB} S R_{l_{1}}$ and $\mathrm{NBC} R_{l_{2}}$ can be orders of magnitude slower than the $\mathrm{NBN} N_{1}$ classifier.

\subsection{Approximations analysis}

At first glance, the presented NB variants are doomed to unfeasible computational burden. Luckily, all methods can be speeded up significantly using a number of approximations, as discussed next. First, as used in [14], LLE can be seen as a local approximation of the richer LLC, which instead of $k N N$ is defined over the whole data. Also for $S R_{l_{1}}$ the performance is seen to degrade slowly when the labeled features used for the decomposition are reduced to a local neighborhood of sufficient size. In [5] only the top nearest neighbors are kept, reducing the computational burden and causing a drop in time complexity to $O(M D+M k+k D s)$ for solving a single $l_{1}$-minimization. The same trick can be applied for $C R_{l_{2}}$, so the critical part remains the retrieval of the nearest neighbors.

ANN. The computational burden is now finding the $k$-nearest neighbors where $k$ is either $1\left(\mathrm{NB} N N_{1}\right.$ and $\left.\mathrm{NB} I N N\right), 5\left(\mathrm{NBN} N_{5}\right.$ and $\left.\mathrm{NB} L L E\right)$, or $200\left(\mathrm{NB} S R_{l_{1}}\right.$ and $\mathrm{NBC} R_{l_{2}}$ ). A lot of effort has been devoted to sub-linear searching by using the structure of the data, and accurate techniques such as kd-tree or hashing can be used especially when the feature dimensionality is low. In our case, the speedup over linear search is small with such approaches. Therefore, we employ approximated nearest neighbors. Here, we use the FLANN implementation [13]. At the price of lower accuracy (out of $k$-nearest neighbors, on average only $90 \%$ are accurately retrieved), we gain a substantial speedup. ANN was also used in $[1,4]$.

Asymmetry. A further improvement in running time of the NB variants can be obtained by sampling less densely. We experiment with an asymmetric scheme where we lower the sampling density on the training material (reducing $M$ ), or on the testing material (reducing $N$ ). Usually, the speedup obtained is equal to the subsampling ratios (except if combined with ANN, when it is significantly lower than the subsampling ratio in training material). [3] suggested reducing 
the sampling density and learning a metric in feature space to compensate for the loss in accuracy. [4] shows that reducing the sampling density in the query usually brings smaller than expected accuracy loss. We arguably show that it is better to keep as dense as possible the features on the query, exploiting all the information available in the query image. Reducing the sampling density for the known labeled features affects less the performance of the NB variants than sampling less densely on the query image. The reason behind this is: if the set of labeled features is sufficiently large, then it is likely to contain redundant data, and subsampling these features still keeps the overall distribution, while subsampling the query image results in loss of valuable data for classification.

\section{Experimental results}

\subsection{Feature extraction}

In our experiments we use both NIMBLE [12] and dense Gradient Kernel Descriptors (G-KDES) [11]. This choice is motivated by the reported performance of both features, consistently outperforming the more conventional SIFT [17] features. Moreover, the NIMBLE features are richer and achieve higher reported performance than SIFT while being an order of magnitude sparser. For NIMBLE we use the code of [12] and follow exactly the settings as in their paper (100 fixations per image and 500-dimensional feature descriptors). Due to its sparse extraction, the running time of the NB methods with NIMBLE is considerably lower than in the case of dense G-KDES or dense SIFT and it is therefore the first choice for most of the experiments we perform. For G-KDES we follow the settings from [11]: extracting dense G-KDES features over a regular grid with 8 pixels step and $16 \times 16$ pixels patches, after resizing the image to a maximum of $300 \times 300$ pixels. We further lower the dimensionality through PCA to 64 .

We include the weighted relative image coordinates in our feature descriptors, as suggested in [1]. Without them there is a significant drop in performance for NBNN [4]. The image coordinates are normalized to $[0,1]$ by dividing with the width and height of the image and added with an empirically fixed weight of $\sqrt{0.5}$ to the $l_{2}$-normalized feature descriptors. This is equivalent to the value 0.5 used in [12] to weight the square $l_{2}$ distances.

\subsection{Implementation}

In the case of $L L E$, we pick the $5 N N$. This is motivated by [14], where the LLC framework performs best when the neighborhood size is 5 . For solving (6) for the $S R_{l_{1}}$ representation, we use the feature sign search algorithm [15] run only on the top NNs to obtain approximated sparse coefficients. However, if the ratio of nearest neighbors w.r.t. the total number of samples used for solving an approximated $S R_{l_{1}}$ representation is too small the loss in performance is significant [9]. Thus, we use 200-NN for the experiments up to 10000 training samples, $500-\mathrm{NN}$ for up to 50000 and $2000-\mathrm{NN}$ for up to 150000 . Similarly to [5], for the feature 




Fig. 1. Performance vs. training size on Scene-15.

sign algorithm, the parameter $\lambda$ is set to 0.3 , which forces a very sparse representation. In case of $C R_{l_{2}}$, equation (7), we first compute the projection matrix, thus speeding up the subsequent computations. For the experiments with ANN we use [13] and build the data structure offline with a target of $90 \%$ correct neighbors in a $k$-NN query. Note that we are building one data structure for the whole pool of labeled features and we retrieve only once the 200-NN for each query feature. From these we pick the $k$-NN, for $N B N N_{k}$, for each class and, in absence of representatives, we take the largest distance to the retrieved samples. This approach was taken also in $[9,18]$. In [18] the neighborhood size is tuned for best performance with FLANN, calling this method Local NBNN.

\subsection{Scene-15}

Scene-15 is a popular benchmark from [19]. We follow the common experimental settings, with a training partition of 100 images per class. We generate 20 random train/test splits and for each keep the performance as the average over per class accuracies. Finally we compute mean performance and standard deviation.

Performance vs. training size. First, we evaluate the impact of the number of training images per class on the performance using NIMBLE features (see Fig. 1). From Fig. 1 we see that considering more neighbors in the NB decision does not necessarily improve the performance. $\mathrm{NB} N N_{1}, \mathrm{NB} N N_{5}$ and $\mathrm{NB} L L E$ perform similarly. $\mathrm{NBC} R_{l_{2}}$ is the best for 10 to 40 training images, while $\mathrm{NB} S R_{l_{1}}$ takes the lead after 40 images. $\mathrm{NB} I N N$ is on par with $\mathrm{NB} S R_{l_{1}}$.

Performance vs. feature density. In Table 1 we report the accuracies for the NB variants under different uniform sampling densities of NIMBLE features in training and test images. Also the effect of using ANN is evaluated. We report mean classification rates and standard deviation for 20 trials for each setting. The best performance is achieved when sampling with the highest possible density in both training and testing images $\left(78.2 \%\right.$-NBINN,77.8\%-NBS $R_{l_{1}}, 74.3 \%$ $\mathrm{NBN} N_{5}, 74.2 \%$-NBNN,73.7\%-NBCR $R_{l_{2}}, 73.1 \%$-NBLLE). Reducing the density results in some performance loss, yet significantly speeds up all methods. Using only a single NIMBLE feature per image, the accuracy for $\mathrm{NB} S R_{l_{1}}$ and $\mathrm{NBC} R_{l_{2}}$ is still above $30 \%$ and $35 \%$, respectively. This is mostly due to the saliency guided NIMBLE feature extraction and the richness of the descriptor itself. The richer representations greatly improve over the standard $N N_{1}$ espe- 
Table 1. Performance versus asymmetry in train/test and use of ANN on Scene-15.



cially in the lower densities settings. Also note how for lower densities, $\mathrm{NBN}_{5}$ also does improve over the $\mathrm{NB} N N_{1}$.

In an asymmetric setting, the performance decreases much faster when lowering the density in the query as compared to lowering the density for the training images, especially for $\mathrm{NBNN}$. With only one feature per query image, $\mathrm{NBC} R_{l_{2}}$ and $\mathrm{NBS} R_{l_{1}}$ still give a reasonable performance (58\% and $\left.55 \%\right)$ vs. $\mathrm{NBN} N_{1}$ $(39 \%)$. In the reversed case, with one feature per training image, the drop in performance is much lower for all the methods, and $\mathrm{NBC} R_{l_{2}} / \mathrm{NBS} R_{l_{1}}$ still reach $65 \% / 66 \%$. NBINN is on par with $\mathrm{NB} S R_{l_{1}}$ for all the settings.

Performance vs. running time. Note that subsampling can result in enormous speedups, with often acceptable drops in performance. For instance, with $\mathrm{NBS} R_{l_{1}}$ a $100 \times$ speedup is achievable at the price of a $9.1 \%$ drop in performance $(68.7 \%$ vs. $77.8 \%)$. NBINN is $10 \times$ faster than and on par with $\mathrm{NB} S R_{l_{1}}$. The ANN speedup increases with the size of the training, and decreases with feature dimensionality. Relaxing the target, by allowing more incorrect neighbors, lowers the performance but the gain in speed might pay off. One $\mathrm{NB} N N$ experiment on Scene-15 using ANN and 100 NIMBLE features per image, takes less than 3 hours on a 2009 Core 2 Quad machine. $\mathrm{NBS} R_{l_{1}}$ is slower, requiring about 4 hours, using a similar 200-NN retrieval per each feature. Nevertheless, the running time per query image per class is way below 1 second, which corresponds to the NBNN time reported in [1] (using dense SIFT).

State-of-the-art. In Table 2 we compare the performance of the NB classifiers proposed here to state-of-the-art results reported in the literature. All these top methods are learning based, i.e. they require prior training and parameter estimation. The Naive Bayes methods, on the other hand, are learning-free and parameter-free to a large extent. One could tune $\lambda$ for $S R_{l_{1}}$ in equation (6), hoping in a better fit to the feature space used, but we did not try this. NBS $R_{l_{1}}$ and NBINN methods improve over the standard NBN $N_{1}$ using either NIMBLE or G-KDES features. The gain in performance is more than $4 \%$ using $I N N$ representations. The recently proposed, Kernelized NBNN with dense SIFT [4], (a learning based method), has similar performance with the $\mathrm{NB} S R_{l_{1}}$ and $\mathrm{NB} I N N$ methods. Our best performance is achieved by NBSR $R_{l_{1}}$ with G-KDES features and is close in performance to the standard learned method Sparse Coding Spatial Pyramid Matching (78.7\% vs. 80.3\%). Note that kernelizing our methods and 
Table 2. Performance Comparison on Scene-15 and Caltech-101

\begin{tabular}{|c|c|c|c|c|}
\hline \multicolumn{2}{|l|}{ Scene-15 } & \multicolumn{3}{|c|}{ Caltech-101 } \\
\hline Method & 100 images & Method & |15 images & 30 images \\
\hline \begin{tabular}{|l|l} 
& ScSPM+SIFT [5] \\
\end{tabular} & $80.28 \pm 0.93$ & ScSPM+SIFT [5] & $67.0 \pm 0.5$ & $73.2 \pm 0.5$ \\
\hline $\left.\begin{array}{|l|l}\Xi & E M K+K D E S\end{array}\right]$ & 87.5 & छ EMK+KDES [11] & ? & 77.5 \\
\hline LScSPM+SIFT [22] & $\mid 89.75 \pm 0.50$ & GLP [21] & 70.34 & 82.6 \\
\hline$\fallingdotseq$ NBNN\&BoF+SIFT [4] & $85 \pm 4$ & $\leftrightarrows$ NBNN\&phow+SIFT [4] & $\mid 69.2 \pm 0.9$ & $75.2 \pm 0$ \\
\hline NBNN- $f_{2}+$ SIFT [4] & $79 \pm 2$ & LLC+HOG [14] & 65.43 & 73 \\
\hline NB- $N N+$ NIMBLE & $74.2 \pm 1$ & NB- $N N+$ NIMBLE & $70.1 \pm 1$ & $\overline{78.1 \pm 1}$ \\
\hline NB- $N N_{5}+$ NIMBLE & $74.2 \pm 1$ & NB- $N N_{5}+$ NIMBLE & $70.2 \pm 1$ & $78.2 \pm 1$ \\
\hline NB- $S R_{l_{1}}+$ NIMBLE & $77.8 \pm 1$ & NB- $S R_{l_{1}}+$ NIMBLE & $\mid 71.8 \pm 0.8$ & $79.73 \pm 1.1$ \\
\hline NB- $I N N+$ NIMBLE & $78.2 \pm 1$ & $\Leftrightarrow$ NB- $I N N+$ NIMBLE & $\mid 72.1 \pm 1.2$ & $80.29 \pm 1$ \\
\hline NB- $C R_{l_{2}}+$ NIMBLE & $73.7 \pm 1$ & NB- $C R_{l_{2}}+$ NIMBLE & & \\
\hline NB- $L L E+\mathrm{NIMBLE}$ & $74.0 \pm 1$ & NB- $L L E+$ NIMBLE & $70.4 \pm 1$ & $78.2 \pm 1$ \\
\hline NB- $N N+$ G-KDES & $75.1 \pm 1$ & NBNN+SIFT [1] ${ }^{*}$ & $\mid 65.0 \pm 1.1$ & 70.4 \\
\hline NB- $N N_{5}+$ G-KDES & $75.1 \pm 1$ & NBNN+NIMBLE [12]* & || $70.8 \pm 0.7$ & $78.5 \pm 1.2$ \\
\hline NB- $S R_{l_{1}}+\mathrm{G}-\mathrm{KDES}$ & $78.7 \pm 1$ & LocalNBNN+SIFT $[18]^{*}$ & $66.1 \pm 1.1$ & $71.9 \pm 0.6$ \\
\hline NB- $I$ & 79.8 & & & \\
\hline NB- $C$ & & & & \\
\hline NB- $L L E+$ G-KDES & $76.4 \pm 1$ & * indicates results withol & ut backgrou & ind class \\
\hline
\end{tabular}

combining them with bag-of-features based methods as in [4] is likely to increase our results further (at the cost of switching to a learning-based scheme).

\subsection{Caltech-101}

On Caltech-101 [20] we report results both with NIMBLE and G-KDES features. In our evaluation (see Table 2), the performance of the $\operatorname{NB} N N$ classifier with NIMBLE features compares to the one reported in the original paper [12] (note that their result is without considering the background class). All the NB variants achieve comparable or better results than the state-of-the-art. $70.3 \%$ at 15 images per class and $82.6 \%$ at 30 images is the best performance out of the state-of-the-art learning methods, achieved by the GLP method of [21]. NBS $R_{l_{1}}$ with NIMBLE features reaches 71.8\%@15 and 79.73\%@30, and NBINN with NIMBLE features reaches 72.1\%@15 and 80.29\%@30. This is the best result using a single descriptor for Caltech-101 with 15 train images per class, to the best of our knowledge.

\subsection{PASCAL VOC 2007}

The PASCAL VOC 2007 [23] has a much higher variability in shape, pose, and position for the 20 annotated object classes than Caltech-101 or Scene-15. We are using NIMBLE features and report results with different sampling densities in training and test images respectively. We report for NBINN the results from [9] and run only $\mathrm{NBNN}$ and $\mathrm{NB} S R_{l_{1}}$ since the other $\mathrm{NB}$ variants gave lower, less robust performance on the previous datasets.

For this challenge, we need to provide class confidences. The relative ranking among the classes for a specific image query is not sufficient, as some images contain instances of multiple classes. Using directly the score from equation (2) for $\mathrm{NB} N N$ is not beneficial. The scores need to be brought to the same meaning, to be comparable across the image queries and not just for the class decision. To this end, we consider the likelihood ratio between the probability of belonging to the class and the probability of not belonging to the class. This resembles the $f_{2}$ kernel from [4], and gives the following confidence function:

$$
S=\sum_{q \in Q}\left(d_{q}^{c}-d_{q}^{\bar{c}}\right), \quad d_{q}^{\bar{c}}=\sum_{x \in N_{k}^{\bar{c}}(q)}\|q-x\|^{2}
$$


Table 3. Image classification results using PASCAL VOC 2007 dataset

\begin{tabular}{|c|c|c|c|c|c|c|c|c|c|c|c|c|c|c|c|c|c|c|c|c|c|}
\hline thess & & & & & & 15 & car & cat & halr & Cow & tabl & $10 g$ & 11001 & IDIke & erse & plan & necp & sofa & train & tv & ver \\
\hline Best of V & 7.5 & 6 & $\pi$ & 71.9 & 3.1 & 60.6 r & 8.0 & 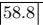 & 53.5 & 42 . & 54.9 & 45. & 77.5 & 64.0 & $\overline{85.9}$ & 36.3 & 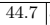 & 60.9 & 79.2 & .2 & 59.4 \\
\hline & 7.2 & .4 & .2 & , & 2.7 & 9.1 & 1.0 & & .5 & 47.9 & 3 & 45.8 & .8 & 67.0 & 4.6 & 0.2 & 4.7 & 3.4 & 9.1 & 3.8 & 0. \\
\hline & 3 & .0 & .8 & & 1 & & & & & & & 27.5 & & & & 2 & 7.1 &  & 0 & & \\
\hline & & & & & & & & & & & & j.t. & & & & & 7.8 & 22.2 & & & \\
\hline & & & & & 2.3 & & & & 42.7 & 9.2 & 8 & 34 & 72.7 & & 0.0 & 8 & 7.1 & 33.8 & 71. & 42 & 0.0 \\
\hline & 2.0 & .2 & \pm & 41 & 2.4 & & 6.1. & 36 & & 18.8 & 20.3 & 28.8 & 62.8 & 45.0 & 6 & 7 & 7.7 & $24.4 \mid$ & 57.7 & 32.8 & 9 \\
\hline$/ 100$ & 7.9 & 3.2 & .1 & 50.7 & 21.6 & & 75.2 & 54.5 & 46.4 & 34.2 & 40.9 & 41. & 73 & 62.1 & 81.4 & 21.1 & 26.9 & 41.9 & 72.7 & 44.9 & 51.2 \\
\hline $\mathrm{NB} S R_{l_{1}}+100 / 1000$ & 67.9 & 63.6 & 47.0 & 50.5 & 22.4 & 57.9 & 75.3 & 56.0 & 47.0 & 34.6 & 37.9 & 41.6 & 74.8 & 62.5 & 81.7 & 21.4 & 28.1 & 43.5 & 73.1 & 46.2 & 51.7 \\
\hline
\end{tabular}

for a given query $Q$ and a class $c$, where $\bar{c}$ is the negative class. The $\operatorname{NBNN}$ method with normalized scores is noted $\operatorname{NBN} N_{S}$, where $k=1$ as in $\operatorname{NBNN}$.

In Table 3 we compare our different settings with the best results from the challenge. When using a strong asymmetry (just 1 feature per training image, 100 features per query image), we already achieve a mean average precision of $37.3 \%$ for $\mathrm{NBN} N_{S}$. Using equal densities (100/100) we obtain a performance of $48.1 \%$, which is $11 \%$ behind the best entry in the VOC2007 challenge. The sparse representations pay off and the $\mathrm{NB} S R_{l_{1}}$ improves over $\mathrm{NB} N N_{S}$ resulting in a mean average precision of $52 \%$ or only $7 \%$ below the top results. This is a good result, taking into account that the best entry in VOC2007 is a learningbased method, more complex than our learning-free approaches. Moreover, we used only the top $200 N N$ to compute the $\mathrm{NB} S R_{l_{1}}$ decision, while $\mathrm{NBINN}$ uses the whole training pool.

\section{Discussion}

Computational costs. The main limitation of NB classifiers is the high computational cost. As shown in Section 3 the time complexity depends (usually) linearly on the number of training images, feature extraction density in training and testing, and feature dimensionality. All these made the deployment of NB $N N$ prohibitive in practice. It remained just a theoretical exercise. Even with exploiting the data structure and using $\mathrm{ANN}, \mathrm{NB} N N$ is quite slow in traditional settings, i.e. for $\sim 5,000$ SIFT features per image and 3,000 training images, it takes $\sim 1$ second per query image and class [1]. Here, we show how to temper the computational costs for NB schemes so they can be used in practice.

Features. The features are the building blocks in NB image classification. Ideally, features should be statistically significant for classification with a low dimensional representation and a low sampling density. The main stream literature [14] heavily relies on the 128 dimensional SIFT features that for high classification performance require uniform sampling densities above 3,000 features per normalized $<0.1$ mega pixels images. The recently proposed NIMBLE [12] features (500 dimensional, 100 sampling density) and G-KDES [11] features (200 dim., $<1000$ density) proved to improve over SIFT based classification and come with (much) lower sampling densities. Using NIMBLE features gives two orders of magnitude speedup over the NBNN SIFT baseline. G-KDES features are also able to severely reduce (one order of magnitude) the running time of a NBNN 
SIFT baseline. The Matlab implementations as provided by $[12,11]$ are running in the range of seconds and can be optimized further.

Training size. The number of training images per class heavily affects the performance. The larger their number the higher the classification rate but at the price of an increase in run time. From Fig. 1 we observe that for up to 5 training images per class, $\mathrm{NBC} R_{l_{2}}$ is the worst performing method, while $\mathrm{NBNN} N_{1}$ and $\mathrm{NBNN} N_{5}$ are best. Collaborative Representation $\left(C R_{l_{2}}\right)$ shows clearest benefit in performance in the middle range (10 up to 40 training images), while after 40 training images $\mathrm{NBC} R_{l_{2}}$ performance scales less well w.r.t. the other $\mathrm{NB}$ variants. When more than 40 training images per class are available, $N B S R_{l_{1}}$ and $\mathrm{NB} I N N$ are clearly the best choices. To the limit, when infinite number of training images are available, it is expected that all NB variants with the exception of $\mathrm{NBC} R_{l_{2}}$ converge in performance. A topic of further research is: how to filter the training features without loss in performance, e.g. by feature selection. Note that from Fig. 1 and Table 1 we see that the number of training images has a bigger impact than the sampling density of the extracted features: e.g. lowering from 100 to 50 the number of training images brings an $8 \%$ decrease in classification rates for $\mathrm{NB} S R_{l_{1}}$, while reducing the feature density from 100 to 50 causes just a $2 \%$ drop. Each training image brings usually something new, while increased sampling over an image is likely to increase the redundancy of the features.

Feature density and asymmetry. What is better: higher sampling density in training or in query images? From Table 1 we see that for all the NB variants there is a higher drop in performance when we uniformly subsample in the query image than when we subsample in the training images. We achieve a 100 fold speedup by extracting a single NIMBLE feature either in each training image (causing a drop of $9 \%$ up to $12 \%$ in performance, depending on the method) or in the query (24\% up to $35 \%$ ). While in training the sampling density is less sensitive, for the query image the subsampling in fact removes important discriminant information for classification. For high feature densities NBS$R_{l_{1}}$ and $\mathrm{NB} I N N$ are our best choices. In very low sampling densities ( $\leq 10$ per image), in either train or query or both, $\mathrm{NBC} R_{l_{2}}$ gets on par or better than $\mathrm{NB} S R_{l_{1}}$ and the other methods - promoting sparsity is less beneficial than using the whole data. It is worth mentioning that in lower densities $\mathrm{NBN} N_{5}$ consistently outperforms $\mathrm{NB} N N_{1}$ by stabilizing the assignments. While not tried, adjusting the neighborhood size to the pool size is expected to improve the results further.

Approximations. A consistent speed up can be obtained by using the structure of the data to drive the NN search. This is especially true when the feature dimensionality is low. Since NIMBLE features are 500 dimensional, we need to use approximated nearest neighbors. We control the chance of accurate neighbor retrieval to $90 \%$, thus the drop in performance is marginal. Note that in our case, we achieve a maximal speedup of $5 \times$. The larger the training pool, the bigger the speedup. Also, the representations can be computed approximatively using a local neighborhood as retrieved using ANN. 
State-of-the-art. Using NIMBLE features instead of SIFT features in a standard setup brings up to two orders of magnitude speed up in a straightforward implementation. This allows us to provide results for large datasets such as PASCAL VOC 2007 (see Table 3) where NB $S R_{l_{1}}$ improves over NB $N N$ adapted for this task. For learning-free methods we show that representations such as $C R_{l_{2}}$, $S R_{l_{1}}$ or $I N N$ are more suitable for high performance than the standard $N N$ under the standard Naive Bayes Image Classification formulation. The methods are validated on Scene-15, Caltech-101, and PASCAL VOC 2007. NBIN N shows improvements over NBSR $R_{l_{1}}$ of $0.5 \%$ on Scene-15 and Caltech-101 (see Table 2) and of $1.4 \%$ on PASCAL VOC 2007 (see Table 3). Moreover, INN while being on par with $S R_{l_{2}}$ it is much faster [9].

While we do not always outperform the state-of-the-art, it is surprising how close we get with our NB variants without any learning stage!

Best practice. For best performance, we suggest the use of NIMBLE features, high sampling densities in the query image (100 features per image), as many different training images as possible (not necessarily highly sampled), ANN for fast query feature neighborhood retrieval and more powerful sparse representation such as $S R_{l_{1}}$ or $I N N$. When the labeled pool is small (tens of images per class) and feature dimensionality is large $(>200), C R_{l_{2}}$ is a good option.

A good tradeoff between speed and performance is given by the following combination: NIMBLE features, low sampling densities (10 per image), ANN, and $\mathrm{NBN} N_{5} / \mathrm{NB} I N N$ for very small training pool of samples, $\mathrm{NBC} R_{l_{2}}$ for a small pool, or $\mathrm{NB} S R_{l_{1}} / \mathrm{NB} I N N$ for large pools.

\section{Conclusions}

In this work we have studied the use of sparse representations in a learning-free, parameter-free setup given by the Naive Bayes Classifier formulation. In particular, the $\mathrm{NB} S R_{l_{1}}$ and $\mathrm{NB} I N N$ give substantial improvements over the standard NBN $N$ approach which has the NN (hard assignment) as basis. Moreover, we have studied asymmetric schemes and the impact of the approximated nearest neighbors on the performance of the NB variants. Combined with recently introduced NIMBLE and G-KDES features, the NBS $R_{l_{1}}$ and NBINN achieves state-of-the-art results for learning-free methods in all the considered benchmarks. Moreover, on Caltech-101, we establish a new state-of-the-art for single descriptor based methods. On PASCAL VOC 2007, we get close to the best entry of the challenge, a learned complex approach. Naive Bayes Classification is still promising. Further directions are to better subsample by feature selection and to explore kernelized versions of the NB variants, learning the priors, and, thus, further improving over our basic methods.

Acknowledgement. This work was partly funded by the Flemish IWT/SBO project ALAMIRE and the ERC research grant COGNIMUND. 


\section{References}

1. Boiman, O., Shechtman, E., Irani, M.: In defense of nearest-neighbor based image classification. In: CVPR, IEEE Computer Society (2008)

2. Behmo, R., Marcombes, P., Dalalyan, A.S., Prinet, V.: Towards optimal naive bayes nearest neighbor. In: ECCV (4). (2010) 171-184

3. Wang, Z., Hu, Y., Chia, L.T.: Image-to-class distance metric learning for image classification. In: ECCV (1). (2010) 706-719

4. Tuytelaars, T., Fritz, M., Saenko, K., Darrell, T.: The NBNN kernel. In: ICCV. (2011)

5. Yang, J., Yu, K., Gong, Y., Huang, T.S.: Linear spatial pyramid matching using sparse coding for image classification. In: CVPR, IEEE (2009) 1794-1801

6. Wright, J., Yang, A.Y., Ganesh, A., Sastry, S., Ma, Y.: Robust face recognition via sparse representation. PAMI 31 (2009)

7. Timofte, R., Van Gool, L.: Sparse representation based projections. In: BMVC. (2011)

8. Roweis, S., Saul, L.: Nonlinear dimensionality reduction by locally linear embedding. In: IEEE ICCV. Volume 290. (2001) 2323-2326

9. Timofte, R., Van Gool, L.: Iterative nearest neighbors for classification and dimensionality reduction. In: CVPR. (2012)

10. Zhang, L., Yang, M., Feng, X.: Sparse representation or collaborative representation: Which helps face recognition? In: ICCV. (2011)

11. Bo, L., Ren, X., Fox, D.: Kernel descriptors for visual recognition. In: Advances in Neural Information Processing Systems. (2010)

12. Kanan, C., Cottrell, G.W.: Robust classification of objects, faces, and flowers using natural image statistics. In: CVPR. (2010) 2472-2479

13. Muja, M., Lowe, D.G.: Fast approximate nearest neighbors with automatic algorithm configuration. In: VISAPP (1). (2009) 331-340

14. Wang, J., Yang, J., Yu, K., Lv, F., Huang, T., Gong, Y.: Locality-constrained linear coding for image classification. In: CVPR. (2010) 3360-3367

15. Lee, H., Battle, A., Raina, R., Ng, A.Y.: Efficient sparse coding algorithms. In Schölkopf, B., Platt, J.C., Hoffman, T., eds.: NIPS, MIT Press (2006) 801-808

16. Timofte, R., Van Gool, L.: Weighted collaborative representation and classification of images. In: ICPR. (2012)

17. Lowe, D.G.: Distinctive image features from scale-invariant keypoints. International Journal of Computer Vision 60 (2004) 91-110

18. McCann, S., Lowe, D.: Local naive bayes nearest neighbor for image classification. In: CVPR. (2012)

19. Lazebnik, S., Schmid, C., Ponce, J.: Beyond bags of features: Spatial pyramid matching for recognizing natural scene categories. In: CVPR (2). (2006)

20. Fei-Fei, L., Fergus, R., Perona, P.: One-shot learning of object categories. IEEE Transactions on Pattern Analysis and Machine Intelligence 28(4) (2006) 594-611

21. Feng, J., Ni, B., Tian, Q., Yan, S.: Geometric lp-norm feature pooling for image classification. In: CVPR, IEEE (2011) 2697-2704

22. Gao, S., Tsang, I.W.H., Chia, L.T., Zhao, P.: Local features are not lonely laplacian sparse coding for image classification. In: CVPR. (2010) 3555-3561

23. Everingham, M., Van Gool, L., Williams, C.K.I., Winn, J., Zisserman, A.: The PASCAL Visual Object Classes Challenge $2007 \quad$ (VOC2007) Results. (http://www.pascalnetwork.org/challenges/VOC/voc2007/workshop/index.html) 